\title{
The Effect on Lower Spine Muscle Activation of Walking on a Narrow Beam in Virtual Reality
}

\author{
Angus Antley and Mel Slater
}

\begin{abstract}
To what extent do people behave in immersive virtual environments as they would in similar situations in a physical environment? There are many ways to address this question, ranging from questionnaires, behavioral studies, and the use of physiological measures. Here we compare the onsets of muscle activity using surface electromyography (EMG) while participants were walking under three different conditions: on a normal floor surface, on a narrow ribbon along the floor, and on a narrow platform raised off the floor. The same situation was rendered in an immersive virtual environment (IVE) Cave-like system, and 12 participants did the three types of walking in a counter-balanced within-groups design. The mean number of EMG activity onsets per unit time followed the same pattern in the virtual environment as in the physical environment significantly higher for walking on the platform compared to walking on the floor. Even though participants knew that they were in fact really walking at floor level in the virtual environment condition, the visual illusion of walking on a raised platform was sufficient to influence their behavior in a measurable way. This opens up the door for this technique to be used in gait and posture related scenarios including rehabilitation.
\end{abstract}

Index Terms-H. Information Technology and Systems, 2. Multimedia Information Systems, b. Artificial, augmented, and virtual realities, d. Evaluation/methodology.

\section{INTRODUCTION}

$\mathrm{W}$ e describe an experiment where participants in an immersive virtual environment (IVE) walked on a virtual narrow raised platform that we call a beam.

When they walked on the virtual beam they exhibited muscle activity that was significantly greater than when they walked on the same narrow path (virtually) located at ground level. Of course all of their walking in the virtual environment really occurred at ground level, only the visual illusion of being above ground realized through the virtual reality displays was responsible for the changes in muscle activity.

An IVE can be characterized by the extent to which participants are able to perceive via normal sensorimotor contingencies [1] - that is, use their body in order to perceive using rules similar to those in physical reality. Normally in physical reality we perceive visually through knowing how to change our gaze direction, for example, by turning our body, head and eyes to enable sight of that which is currently 180 degrees behind us, or moving our head so as to see beyond an object that currently obscures a point of interest, or moving our head closer to something in order to be able to see or hear it more clearly. No virtual reality system in existence today can enable the full range of sensorimotor contingencies (SCs) that are possible in physical reality - for example, constraints on

- Angus Antley is with the Department of Computer Science, University College London. E-mail: a.antley@ cs.ucl.ac.uk.

- Mel Slater is with the Catalan Institute of Research and Advanced Studies (ICREA) at the University of Barcelona. E-mail: melslater@ub.edu.

Manuscript received (insert date of submission if desired). Please note that all acknowledgments should be placed at the end of the paper, before the bibliography. display resolution make close viewing of an object impossible, and the lack of generalized haptics makes the vast majority of haptic perceptual actions impossible in IVEs. Nevertheless, there are systems that approximate this to varying extents - a typical head-mounted display (HMD) with head-tracking allows visual SCs in any direction, but the field of view is often highly constrained and the resolution is orders of magnitude less than natural vision. A multi-wall stereo projection system with tracking, such as a Cave $[2,3]$ also supports an approximation to at least visual SCs, but again, a Cave is highly constrained, though usually with higher resolution than a HMD.

Participants in an immersive virtual environment will typically experience the illusion of being in the place depicted by the displays. We refer to this as Place Illusion (PI) [4]. This has usually been referred to in the literature as 'presence' [5-10], derived from the term 'telepresence' [11], the feeling that people operating a teleoperator system might have of being at the remote site of the robot. The term 'presence', however, has come to be overloaded with many different possible meanings that go beyond the original concept of the strong illusion of being in a place that is associated with perceiving a remote or virtual space through natural SCs. Therefore we reserve the term PI to refer specifically to the strong illusion of being in the virtual place.

A major hypothesis underlying much of our research in this field over several years is that when PI occurs and when situations and events depicted in the virtual environment belong to a possible and plausible world (not necessarily a physically realizable world) that participants 
will tend to respond to their virtual experience as if it were real. This response-as-if-real (RAIR) occurs because not only is the situation being depicted a possible one, but the participant is personally 'there' as part of it. At some level the brain does not distinguish between reality and virtual reality, and produces automatic subjective, behavioral and physiological responses that correspond to the hypothesis: 'this is really happening, and it is happening in my vicinity'. Of course, participants know for sure that what is happening is not real, and that they are not in the virtual place, but we are referring here to those immediate and automatic responses that occur before conscious reflection, and which conscious reflection does not impede.

There is substantial evidence that RAIR occurs in a number of specific domains. For example, it has been shown that higher anxiety is reported when people speak to a group of negatively behaving virtual characters compared to a neutral or positively behaving audience [12]. A visual cliff type of environment has been used where participants were in an IVE that shows a precipice, and their heart rate increased significantly [13-15]. Both skin conductance, heart rate and heart rate variability have been shown to respond significantly in the context of general social situations [16], and social situations that are highly stressful [17]. It has also been found that there are similarities in behavior when participants play handball in virtual reality compared to physical reality [18]. In some of these examples anxiety, as measured through physiological responses, is one sign that people are responding to the virtual events as if they were really happening. Eye scanpaths have also been demonstrated to change appropriately within an IVE experience [19].

\section{Hypothesis}

Our experiment was designed to test the hypothesis that the increase in muscle activation in response to walking on a reduced area of support above ground level when compared to walking at ground level can be induced within an IVE. Moreover, a comparison is made with the same setup in physical reality. In a real room experimental participants exhibited more frequent muscle activations of the extensor muscles of the lower spine when walking on a narrow, raised platform than they did walking on the floor. This prevented their centre of gravity from falling outside the narrowed base of support afforded by the platform. Our hypothesis was that when the participants would perceive the raised platform while walking in an IVE modeled on the physical room and platform they would exhibit a similar proportional increase in these muscle activations when compared to walking on the virtual floor.

In previous research the focus of attention has been on subjective responses as elicited by questionnaires, and autonomic nervous system physiological responses such as heart rate. Here we show that there are measureable muscular changes in the act of walking within an IVE as a function of the type of the area of support that is depicted. Moreover, the direction of changes in muscle activity is the same as those in a similar physical reality, even if the absolute levels are not the same. In Section 2 we describe the materials and methods of the experiment, including the design, scenario, equipment, and method of analysis. In Section 3 we give the results, followed by discussion and conclusions in Sections 4 and 5. The experiments described were approved by the UCL Ethics Committee.

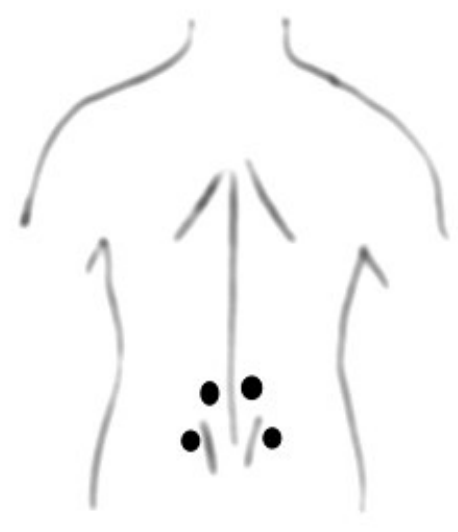

Fig. 1. The placement position of the EMG sensors on either side of the lower spine.

\section{Materials and Methods}

\subsection{Materials}

The experiment was conducted in two locations. The first was a Trimension ReaCTor, which is a Cave-like projection based system. The second location was a nearby room in our laboratory. The Trimension Reactor system has three $3 \mathrm{~m} \times 2.8 \mathrm{~m}$ back-projected screens: front, left, and right, and a $3 \mathrm{~m} \times 3 \mathrm{~m}$ front projection surface on the floor. The system is controlled by a Windows based PC cluster. The computers in the cluster contained Intel Pentium 3.2 GHz processors with 1 gigabyte of RAM and Nvidia Quadro FX 5600 graphics cards. The participants were fitted with shutter glasses that were synchronized with the projectors delivering active stereo at $45 \mathrm{~Hz}$ each eye. Attached to the top of the glasses was an InterSense IS-900 tracking device to track the head of the participant. Also, each participant was fitted with a Mind Media Nexus-4 wireless physiological device that recorded two channels of surface electromyogram (EMG) at $1024 \mathrm{~Hz}$. For each channel two electrodes were attached to the left and right of the lumbar spine parallel to the L4 and L5 vertebrae, as shown Fig. 1.

\subsection{EMG Data Processing}

The dependent variable for this experiment was the number of muscle activations or onsets that were extracted from the surface EMG of each participant in each of the six conditions per unit time. Other things being equal we would expect a rise in number of extracted onsets in the beam compared to the floor conditions. 
Briefly, an onset occurs when the filtered and rectified EMG signal stays above a threshold value for more than $25 \mathrm{~ms}$. This threshold value is computed as three times the standard deviation of a baseline signal. In other words an onset is a period of atypically high activity. This method is based on that described in [20].

In our experiment EMG was recorded for the erector spinae muscles of the lumbar spine using the Nexus-4, which samples at $1024 \mathrm{~Hz}$ and a signal to noise ratio of $53.5 \mathrm{~dB}$ for the reference signal of $1 \mathrm{mV}$ at 32 hertz. The differential sensors have a Common Mode Rejection Ratio (CMRR) of $100 \mathrm{~dB}$. We now describe in more detail how the onsets were computed.

The Nexus-4 device has anti-alias hardware filters to remove all frequencies above $450 \mathrm{~Hz}$. De Luca [21] suggests that the full bandwidth for the surface EMG signal lies between $20 \mathrm{~Hz}$ and $500 \mathrm{~Hz}$. Therefore, we used a $3^{\text {rd }}$ order Butterworth filter to high pass filter the raw EMG data above $20 \mathrm{~Hz}$. Next, the resulting signal was rectified by taking the absolute value. We then low pass filtered the resulting data below $50 \mathrm{~Hz}$ following the method of [20].

In [20] the threshold for the onsets was computed in order to find the delays for activity onsets for muscles when subjects were asked to deliberately activate those muscles. In those conditions the baseline period for computing the threshold was taken as a $50 \mathrm{~ms}$ period while the muscle was 'resting'. This period is fine for identifying an initial use of a muscle against a background of resting, but is not appropriate for extracting onsets from the background noise of walking. Since in this experiment we were counting the number of onsets that occurred in a given time period, and since we were interested in the comparisons of these counts between different walking conditions, we therefore used the whole signal from the 'walking on the floor' condition as our baseline. Therefore the standard deviation from this data for each participant was computed, and the threshold defined as three times the standard deviation.

In order to then identify the number of offsets in a signal we counted the number of peaks in the filtered rectified data that stayed above the threshold for the duration of at least 25 milliseconds. This is shown in Fig. 2. This threshold and duration were used to compute the number of onsets for each of the six conditions. For each participant we then had onset counts for the left and right sensors for each condition; a total of twelve values.

\subsection{VIRTUAL ROOM MODEL}

The model for this experiment was built using the Blender 3D content creation platform. The model was built to match exactly the dimensions of the room in our laboratory (Fig. 3). The lighting model was implemented using 3D Studio MAX. In this model the reflections were viewpoint independent.

Static objects in the scene had shadows that were baked into the textures used in the model. The shadows in this lighting model provided the participants with further confirming information that the virtual platform was raised. The model was rendered on the PC cluster using the XVR platform software [22]. The light levels in the virtual and the real room were matched using a Precision Gold lux and light meter that was accurate to within 0.1 lux. It should be noted that, while average light levels were matched, the contrast levels were still different. This meant that the real room was less dim in areas near the light sources than the virtual room.

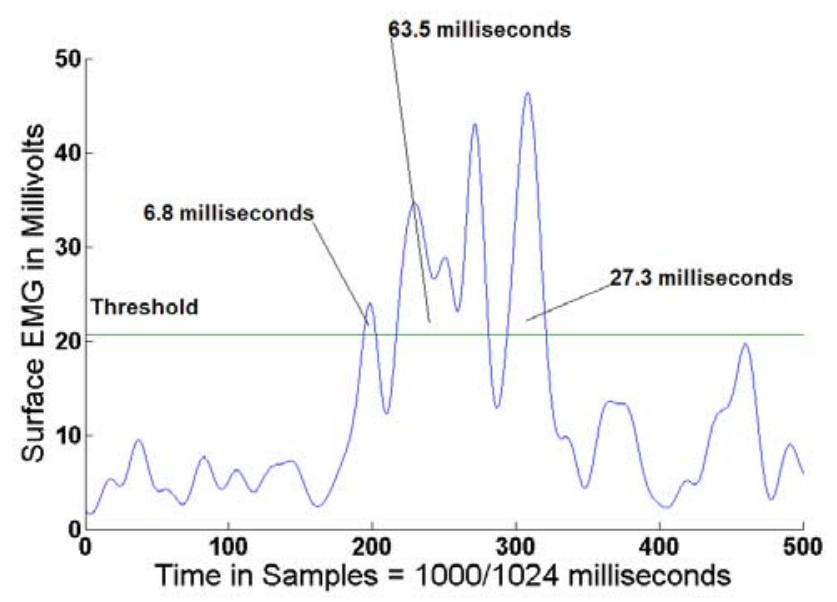

Fig. 2. The rectified filtered signal above shows the detection of two onsets, the first peak crosses the threshold for less than 25 milliseconds and is therefore not counted as an onset.
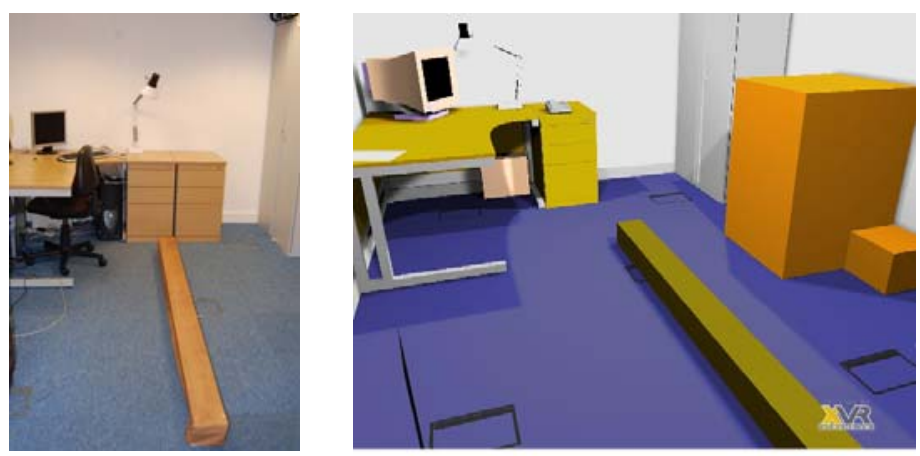

Fig. 3. (a) The real room (left) and (b) the virtual room (right).

\subsection{Experimental Design}

There were 12 male participants in the experiment in a within-groups design, where each experienced the Real Room (RR) and Virtual Room (VR), both of which had three levels. The participants were recruited using a mass email amongst the University College London community. All the participants were healthy males with the mean and standard deviation of age being $25 \pm 13$ years.

The overall design is shown in Table 1. Six of the participants experienced the RR first and then the VR, and the remainder in the other order. The three conditions in each group were (1) walking across the unmarked floor, (2) the same walk 
except that the floor was marked by a strip of material (real or virtual) and (3) the same walk except that the participants were on a narrow platform (real or virtual). There are six possible orders of the three conditions, and in each group participants were assigned randomly to the conditions.

\section{TABLE 1}

\section{CONDITIONS OF THE EXPERIMENT}

\begin{tabular}{|l|l|}
\hline \multicolumn{1}{|c|}{ REAL ROOM } & \multicolumn{1}{c|}{ VIRTUAL ROOM } \\
\hline (RRf) Walking on the Floor & (VRf) Walking on the Floor \\
\hline (RRs) Walking on the Strip & (VRs) Walking on the Strip \\
\hline (RRb) Walking on the Beam & (VRb) Walking on the Beam \\
\hline
\end{tabular}

Each of the participants in the experiment were required to walk a distance of $235 \mathrm{~cm}$ out and back ten times in each of the three real and three virtual conditions. In the beam condition participants did not step off the beam, but turned in place (in both the real or virtual conditions). The room has white walls and a blue carpeted floor, a desk, a chair and a lamp (Fig. 3). The strip of material flat on the floor in condition 2 was $235 \mathrm{~cm} \times 14 \mathrm{~cm}$. The platform in condition 3 had length $235 \mathrm{~cm}$, width $14 \mathrm{~cm}$ and height $13 \mathrm{~cm}$.

In the VR conditions 1 and 2, the floor of the environment was registered to the same level as the real floor of the Trimension. In VR condition 3 the top of the platform was registered to the level of the floor of the Trimension. Therefore the virtual floor was $13 \mathrm{~cm}$ below the level of the real floor.

\subsection{Procedures}

Upon arrival at the laboratory, each participant was given a pre-experiment questionnaire. This recorded the relevant background information for each participant, including age, video game experience and back pain history. After completing the questionnaire, participants were given a handout describing the procedures of the experiment. This included a warning of the possible side effects from experiencing virtual environments, such as nausea and epileptic episodes. When they had read the information sheet, the participants were given the option of withdrawing from the experiment. None of the participants withdrew at this point, but instead signed a standard consent form.

After agreeing to continue, the EMG sensors were placed on the skin along each side of the participant's lower lumbar spine. After the EMG sensors were attached, each participant had the task explained to them. The Nexus- 4 device was turned on and the server that recorded the EMG data was started.

The participants were assigned to their factor order (VR or RR first), followed by platform or floor or strip order within the VR or the RR. In all conditions the participants were asked to walk out and back, the distance of 2.35 meters, ten times, of course on the strip or platform in those conditions. The participants were also instructed to remain in a state of quiet standing for one minute before each condition began. A video recorder was then started. In the VR condition the participants had their shoes covered in order to avoid marking the floor (itself a screen).
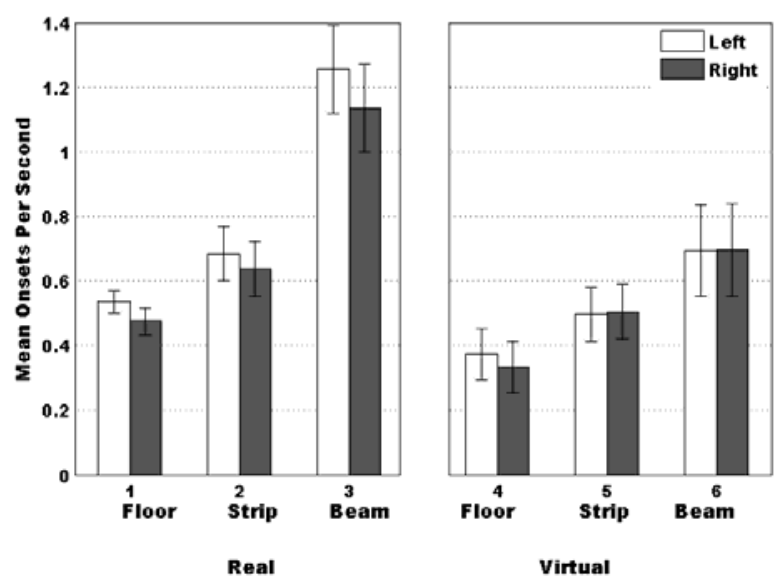

Fig. 4. The mean number of onsets per second for each condition. The whisker represents the standard error for the data for that condition.

Between each trial in the Cave (Trimension) the participants were told to look at a floor box cover at the right end of the virtual platform. This was to ensure that they saw the change in height for the virtual platform condition. Also, in the VR conditions events were recorded to indicate when the participant walked back to their starting point. This allowed us to remove from our analysis the data recorded during the time that the participant lost stereo vision in the Cave because they would have been facing the side of the Cave that did not have a screen.

At the end of the final condition, the video and the physiological recording were stopped and each participant was asked the question, "Did you see a raised platform in any of the Cave conditions?" (The answers were all 'yes'). The participants were then paid 7 GBP and asked not to discuss this experiment with anyone for at least three months.

\section{Results}

In this experiment we were interested in comparing how people walked, as measured by the EMG onset activations, in the real and virtual conditions, and in particular whether the narrowed base of support afforded by a virtual platform would induce a similar balance reaction as that of a real platform. The balance reaction was determined by the number of onset activities in the erector spinae muscles of the lumbar spine.

Fig. 4 shows bar graphs of the mean number of onsets per second in each condition. It is clear that for both the VR and RR conditions that there is a significant difference in mean number of onsets between the platform and the floor, but that the absolute levels are greater for the RR in the 
beam condition.

The Condition and Subject were the independent variables. Recall that Condition has 6 levels; for the real room walking on the unmarked floor (RRf), walking on a strip (RRs), and walking on a beam (RRb), and similarly another 3 conditions for the virtual environment - VRf, VRs, and VRb. Two-way analyses of variance (ANOVA) were carried out with the log of number of onsets per second as the response variable (separately for the left and right sides). The log response was taken since initial analyses revealed that the residuals of the fit did not have a normal distribution. 'Subject' was included as independent factor to take account of inter-subject variability. 'Order' (whether the VR condition was experienced before or after the RR condition) had no effect and is not considered further here.

For the left onsets the ANOVA revealed a significance difference between the 6 conditions $\left(\mathrm{P}<3.3 \times 10^{-7}\right)$. Multiple contrast analysis with an overall significance level of $\mathrm{P}=$ 0.05 results in:

$$
\mu_{R R f} \approx \mu_{R R s}<\mu_{R R b}
$$

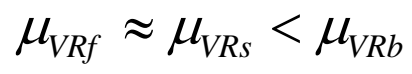

where $\approx$ means 'not significantly different from' and $<$ means significantly less than.

For the right onsets there is a significant difference between the conditions $(\mathrm{P}<0.003)$, and the multiple comparisons tests reveal exactly the same qualitative results as above.

In each case a Jarque-Bera test [23] did not reject the hypothesis of normality of the residuals $(\mathrm{P}=0.19$ and 0.34 for the left and right ANOVAs respectively). In each case one of the 72 readings was removed due to the response being an extreme outlier for both the left and right onsets. These readings, belonging to the same participant, were excluded from the results above.

Comparing the RR with the VR means using multiple contrasts, for the left and right onsets, only the beam RR condition for the real walking is significantly higher than the corresponding VR condition.

For the left onsets there were significant inter-subject variations ( 3 subjects had mean number of offsets that were significantly less than some of the remainder with respect to condition VRf). If these subjects are removed then the result for the VR condition in (1) holds for $\mathrm{P}<0.1$ but not for $\mathrm{P}<0.05$. For the right onsets one subject has mean significantly lower than the remainder, and when removed the results (1) remain unchanged.

\section{Discussion}

Overall the results might usefully be framed in the context of a Bayesian model of action, where the probability that the world is in a given state is some function of prior belief about the state of the world and our current sensory input. The action taken is a function of the probability of the current state and the consequences of the action [24]. The increase in extensor muscle activations on the platform is an action that reduces the probability of falling in the RR case. The results of the experiment suggest that this also occurs, though with a lesser intensity in the VR case. The visual sensory information in the VR is convincing enough, despite the lack of haptic feedback, to make the probability that the person is walking on a raised platform sufficiently above zero for it to affect measurable behavior. This probability combined with our knowledge of the consequences of falling induces the bracing action.

In previous experiments physiological measures have been used to identify stress reactions that were induced by a virtual environment. These stress reactions have been presented as evidence that the virtual display has been accepted as a substitute for reality by the participant (hence there is RAIR). It could be said that someone watching a movie could have a similar stress reaction. The difference here is the use of whole body sensorimotor contingencies, the use of the whole body in walking as normal - clearly impossible when playing a traditional computer game or watching a movie. Sitting in a chair, muscles are not stretched and stretch reflexes are not activated as they would be in real walking. Peripheral vision, as can only be simulated in a surrounding virtual display, is crucial for determining the correct response to keep a person from falling.

The results of this paper therefore also help to delineate the differences between the experience of a virtual environment through a desktop interface and immersive systems that inevitably engage the whole body in movement. The issue of engaging the whole body for locomoting through a VR and its positive influence on the reported sense of presence has been shown in a previous questionnaire study [25]. Since we are evaluating an everyday activity such as walking, this result also holds promise as a first step towards establishing what a valid response to a non-stressful virtual environment might look like. Also it holds the promise that IVR might be used successfully in the understanding and treatment of postural and gait related pathologies, for example [26, 27].

\section{Conclusion}

In this paper we have described an experiment that used EMG recordings to present evidence that people experiencing an immersive virtual reality react to the stimulus of the virtual environment rather than the real world in which the whole experience is, of course, embedded. Having established that EMG can detect the response of a participant's body to a virtual world, the next step would be to further investigate the sensitivity of EMG to more subtle variations in the conditions. This means that rather than changing the overall content of the scene between conditions, we may change just the sophistication of the lighting by adding dynamic shadows or improving the physics to allow the user to 'step up' on to the virtual platform. In order for designers of virtual reality systems to use such a measure in practice it must be shown to be 
sufficiently sensitive to such differences in configuration.

It should be noted that in spite of the similarity between the RR and VR conditions in changes in the muscle activation between floor, strip and beam there is a large and significant difference between activation on the beam in RR and the beam in VR. Anecdotally, this was visible to the experimenters who saw that when participants would reach the end of the real beam, they would exhibit greater body sway to keep their balance on turning. It is clear that when a person's foot moves beyond the edge of the platform, they will experience an absence of supporting thrust that confirms the fact that the participant is at height. We have little doubt that this haptic feedback accounted for some of the difference between the real and virtual beam conditions. Further work must attempt to understand these differences, whether due only to cognitive reasons (participants know in VR that they are not on a beam), or a failure in haptics (they do not feel as if they are walking on a wooden beam), or due to visual perception (the difference in the quality of visual images). Much research remains to be done in this area.

\section{ACKNOWLEDGMENT}

This research was funded under the EU FET Integrated Project PRESENCCIA Contract Number 27731. Thanks to Dr Henrik Ehrsson and Dr Robert Leeb for helpful comments on this paper.

\section{References}

[1] A. Noë, Action In Perception: Bradford Book, 2004.

[2] C. Cruz-Neira, D. J. Sandin, T. A. DeFanti et al., "The CAVE: audio visual experience automatic virtual environment," Communications of the ACM, vol. 35 , no. 6 , pp. 64-72, 1992.

[3] C. Cruz-Neira, D. J. Sandin, and T. A. DeFanti, "Surroundscreen projection-based virtual reality: the design and implementation of the CAVE," Proceedings of the 20th annual conference on Computer graphics and interactive techniques, pp. 135-142, 1993.

[4] M. Slater "Place Illusion and Plausibility Can Lead to Realistic Behaviour in Immersive Virtual Environments," Philos Trans R Soc Lond, vol. in press, 2009.

[5] R. M. Held, and N. I. Durlach, "Telepresence," Presence: Teleoperators and Virtual Environments, vol. 1, no. 1, pp. 109-112, 1992.

[6] W. Barfield, and S. Weghorst, "The Sense of Presence Within Virtual Environments: A Conceptual Framework," Human-Computer Interaction:Software and Hardware Interfaces, G. Salvendy and M. Smith, eds., pp. 699-704: Elsevier Publisher, 1993.

[7] T. B. Sheridan, "Musings on Telepresence and Virtual Presence," Presence: Teleoperators and Virtual Environments, vol. 1, no. 1, pp. 120-126, 1992.

[8] T. B. Sheridan, "Further musings on the psychophysics of presence," Presence: Teleoperators and Virtual Environments, vol. 5, no. 2, pp. 241-246, 1996.

[9] J. V. Draper, D. B. Kaber, and J. M. Usher, "Telepresence," Human Factors, vol. 40, no. 3, pp. 354-375, 1998.
[10] M. V. Sanchez-Vives, and M. Slater, "From Presence to Consciousness through Virtual Reality," Nature Reviews Neuroscience, vol. 6, no. 4, pp. 332-339, 2005.

[11] M. Minsky, "Telepresence," Omni, pp. 45-52, 1980.

[12] D. P. Pertaub, M. Slater, and C. Barker, "An experiment on public speaking anxiety in response to three different types of virtual audience," Presence-Teleoperators and Virtual Environments, vol. 11, no. 1, pp. 68-78, 2002.

[13] M. Meehan, S. Razzaque, M. Whitton et al., "Effect of latency on presence in stressful virtual environments," Virtual Reality, 2003. Proceedings. IEEE, pp. 141-148, 2003.

[14] M. Meehan, B. Insko, M. Whitton et al., "Physiological measures of presence in stressful virtual environments,"

Acm Transactions on Graphics, vol. 21, no. 3, pp. 645-652, 2002.

[15] M. Slater, P. Khanna, J. Mortensen et al., "Visual Realism Enhances Realistic Response in an Immersive Virtual Environment," IEEE Computer Graphics and Applications, vol. 29, no. 3, pp. 76-84, 2009.

[16] M. Slater, C. Guger, G. Edlinger et al., "Analysis of Physiological Responses to a Social Situation in an Immersive Virtual Environment," Presence: Teleoper. Virtual Environ., vol. 15(5), pp. 553-569, 2006.

[17] M. Slater, A. Antley, A. Davison et al., "A virtual reprise of the stanley milgram obedience experiments," PLOS ONE, vol. 1, no. 1, pp. e39. doi:10.1371/journal.pone.0000039, Dec 20, 2006.

[18] B. Bideau, R. Kulpa, S. Menardais et al., "Real handball goalkeeper vs. virtual handball thrower," PresenceTeleoperators and Virtual Environments, vol. 12, no. 4, pp. 411-421, 2003.

[19] J. Jordan, and M. Slater "An Analysis of Eye Scan Path Entropy in a Progressively Forming Virtual Environment," Presence: Teleoper. Virtual Environ., vol. 18, no. 3, pp. 185-199, 2009.

[20] R. Di Fabio, "Reliability of computerized surface electromyography for determining the onset of muscle activity.," Phys Ther, vol. 67, no. 1, pp. 43-8, 1987.

[21] C. De Luca, "The Use of Surface Electromyography in Biomechanics," JOURNAL OF APPLIED BIOMECHANICS, vol. 13, pp. 135-163, 1997.

[22] M. Carrozzino, F. Tecchia, S. Bacinelli et al., "Lowering the development time of multimodal interactive application: the real-life experience of the XVR project." pp. 270-273.

[23] A. Bera, and C. Jarque, "Efficient tests for normality, homoscedasticity and serial independence of regression residuals: Monte carlo evidence," Economics Letters, vol. 7, no. 4, pp. 313-318, 1981.

[24] K. P. Kording, and D. M. Wolpert, "Bayesian integration in sensorimotor learning," Nature, vol. 427, no. 6971, pp. 244-247, JAN 15, 2004.

[25] M. Usoh, K. Arthur, M. C. Whitton et al., "Walking > walking-in-place > flying, in virtual environments." pp. 359364.

[26] H. Sveistrup, "Motor rehabilitation using virtual reality," Journal of NeuroEngineering and Rehabilitation, vol. 1, no. 1, pp. 10, 2004. 
[27]

M. K. Holden, "Virtual environments for motor

rehabilitation: review," Cyberpsychology \& behavior, vol. 8,

no. 3, pp. 187-211, 2005.

Angus Antley is a Research Fellow in the Department of Computer Science, University College London, where he is carrying out research on body representation and posture in immersive virtual environments. He obtained his AB in English from Duke University in 1989, and an MS in the Department of Computer Science, University of North Carolina at Chapel Hill in 2002. Contact him at a.antley@cs.ucl.ac.uk.

Mel Slater is a research professor at the Catalan Institute for Research and Advanced Studies (ICREA) at the University of Barcelona, Spain, and a professor of virtual environments at University College London in the department of Computer Science. His research interests include global illumination algorithms for virtual reality and scientific understanding of how people respond to virtual reality. Slater has a DSc from the University of London. Contact him at mel.slater@ub.edu. 